\title{
DETERMINATION OF FORAGE PRODUCTIVITY, CARRYING CAPACITY AND PALATABILITY OF BROWSE VEGETATION IN ARID RANGELANDS OF CHOLISTAN DESERT (PAKISTAN)
}

\author{
ABDULLAH, M. $^{{ }^{*}}{ }^{*}$ - RAFAY, M. $^{2}$ - SIAL, N. ${ }^{3}$ - RASHEED, F. ${ }^{4}$ - NAWAZ, M. F. ${ }^{4}-$ \\ NOUMAN, W. $.^{5}-$ AHMAD, I. ${ }^{4}-$ RUBY, T. $^{3}-$ KHALIL, S. $^{2}$ \\ ${ }^{I}$ Cholistan Institute of Desert Studies, The Islamia University of Bahawalpur, Pakistan \\ ${ }^{2}$ Department of Forestry, Range and Wildlife Management, The Islamia University of \\ Bahawalpur, Pakistan \\ ${ }^{3}$ Department of Life Sciences, The Islamia University of Bahawalpur, Pakistan \\ ${ }^{4}$ Department of Forestry and Range Management, University of Agriculture Faisalabad, \\ Pakistan \\ ${ }^{5}$ Department of Forestry and Range Management, Bahauddin Zakariya University, \\ Multan, Pakistan \\ *Corresponding author \\ e-mail: abdullahfrw@gmail.com \\ (Received $3^{\text {rd }}$ Apr 2017; accepted $26^{\text {th }}$ Jun 2017)
}

\begin{abstract}
The browse vegetation in Cholistan rangelands of Pakistan have been on decline due to climatic extremes, overgrazing and anthropogenic pressure. Study area is hot arid desert where vegetation grow after rainfall but over grazing, extreme weather, and local comunity pressure for fire wood, timber, and harvesting of plants for varius purposes causing the decline of browse species. Therefore, a study was carried to investigate forage productivity, carrying capacity and palatability of browses. Multiple surveys were conducted from 2011-2012 at twenty range stands and 25 browse species belonging to 12 families were identified. Results revealed that browse productivity was high $(8029.1 \mathrm{~kg} / \mathrm{ha})$ in wet season as compared to dry season $(5422.9 \mathrm{~kg} / \mathrm{ha})$, correspondingly carrying capacity was high during wet season $(16 \mathrm{ha} / \mathrm{AU} / \mathrm{Y})$ than dry season $(24 \mathrm{ha} / \mathrm{AU} / \mathrm{Y})$. Moreover, during dry season, mostly stands were observed to be overgrazed while in wet season maximum stands were moderately grazed. High carrying capacity and good grazing status of stands in wet season was due to better forage production. Based on palatability classification, 22 species were found to have palatability to varying degree and 3 species were nonpalatable. In palatable species, leaves of 14 species; shoot/stem of 13 species, flower of 4 species, and fruit of 3 species were grazed by livestock, whereas cattle were observed to graze on 7 species; goat and sheep like 10 species each while camel prefer 20 species. The findings of this study indicate that overall the browse productivity of Cholistan rangelands was low and vary according to seasonal rainfall. Therefore, protection, suitable stocking rate and planned grazing is vital to conserve browses ultimately for sustainability of Cholistan rangelands.
\end{abstract}

Keywords: browse species, biomass, stocking rate, grazing livestock, sustainability

\section{Introduction}

Rangelands cover about $50 \%$ of the world's land surface and are large tracts of natural vegetation which support livestock. Mostly rangelands are in vegetation biomes such as grasslands, shrublands, savannas, and deserts. These areas are often characterized by arid climate with low rainfall, and seasonal temperature extremities. Studying the relationship among rangelands components (livestock and plants) is most important tools to adopt suitable measures for best practical use of range resources 
(Friedel et al., 2000). Rangelands provide vegetation cover and soil protection which ensures sustainable production of forage for livestock. Especially browses (shrubs and tree foliage) beside grasses compose cheap sources of feed for animals in World. The importance of browse species as animal feed is reported especially from harsh environments in arid and semi-arid rangelands. Mostly these species have advantage of maintaining their nutritive value and greenness during dry season when grasses decline in both quantity and quality. This nutritious profusion and perennial performance of browses afford round the year provision of forage for livestock (Mtengeti and Mhelela, 2006).

Rangelands quality largely dictate animal productivity and thus it becomes vital to maintain sustainable feed resources from rangelands without deterioration (Rubanza et al., 2006). The justifiable use of rangelands is vital for the development of national economy. Pakistan is a sub-tropical country, which consists of vast semi-arid and arid tracks of land, stretches over 68 million hectares (Majeed et al., 2002). Pakistan has a wealth of 135 million heads of livestock, which account for $10.8 \%$ of the GDP. Livestock sector play very significant role because it provides numerous services for humankind like milk and meat, which are vital components of our diet. Livestock occupies a key position in the rural economy of Pakistan for improving the living standard of small resource peoples (Khan et al., 2005).

The rangelands are degrading due to overgrazing, unscientific livestock management, excessiveness of unpalatable species, change in climate, and disturbance of soil (Landsberg and Crowley, 2004). Previous policies have always supported the crops production over livestock, leading in misuse of lands having economically ineffectual productive potential. The herbaceous vegetation of these rangelands only flourishes in monsoon season; accordingly, livestock herds show pitiable health and produce very poor yield of meat and milk. These problems are common everywhere in the world where arid or semiarid rangelands exist. Therefore, developing countries like Pakistan face the similar situation in their rangelands health. Rangelands, which constitute about $65 \%$ of the total area of Pakistan, are in declining process with the passage of time (Ahmad and Hasnain, 2001).

Furthermore, Loeser et al. (2006) showed that episodic drought interacts with grazing, leading to infrequent but biologically important shift in plant communities and suggested the importance of climatic variation in determining ecological effects of grazing practices. Few studies have attempted to unravel the effects of grazing and drought on desert rangelands. It appeared that negative effects of grazing on natality, mortality, or population turnover can be accentuated for certain species when subject to below average precipitation (Curtin, 2002). Globally, grazing has been one of the key disturbance factors resulting in rangeland degradation, an increase of spatial homogeneity of the rangelands, an alteration of rangelands function, and loss of species diversity (Gamoun, 2014).

The Cholistan rangelands were formerly a thriving and prosperous area but now largely converting into an abandoned patch. The productivity of its rangelands is degrading ultimately, carrying capacity of this area is decreasing (Akhter and Arshad, 2006). Sustainability of life in this hot desert rotates around the annual rainfall. During summer season, weather is tremendously severe and harsh; certain xeric plant species survive but suffer high grazing pressure and leading to partial eradication (Arshad et al., 2008). Resultantly, the palatable species are diminishing and unpalatable species with less nutritious properties are becoming abundant. Continuous increase in human 
population for livelihood and multiplying number of livestock is adding towards the desertification (Abdullah et al., 2013).

Assessing forage productivity and carrying capacity are key factors of rangeland inventory and monitoring programs which are highly required for sustainability of natural resources. The browse species are one of the most important and nutritionally rich sources of feed for livestock in Cholistan rangelands. Due to year-round stress, the browses of Cholistan rangelands are under severe threat and need detail assessment of their potential. Already no conservational measures have been made in Cholistan rangelands because of unavailability of sufficient data. In order to preserve the optimum production of browse species and their justifiable use in future, information about current range resources is very important. Therefore, this study was being planned to collect base line data about productivity of browses, carrying capacity and palatability to chalk out their management strategy in Cholistan rangelands.

\section{Material and methods}

\section{Description of study area}

The study was conducted in Cholistan desert of Pakistan. Cholistan desert, an extension of the Great Indian Desert, is located in southern Punjab of Pakistan, between $27^{\circ} 42^{\prime}$ and $29^{\circ} 45^{\prime}$ north and $69^{\circ} 52^{\prime}$ and $73^{\circ}$ 05' east (Fig. 1). The climate of Cholistan desert is characterized by low and sporadic rainfall. The mean annual rainfall varies from less than $100 \mathrm{~mm}$ in the west to $200 \mathrm{~mm}$ in the east. Rain usually falls during monsoon (July through September), winter and spring (January through March). Aridity is the most striking feature of the Cholistan desert with wet and dry years occurring in clusters. Cholistan is one of the hottest regions of Pakistan. Temperatures are high in summer and mild in winter. The mean summer temperature (May, June) is $34^{\circ} \mathrm{C}$ with the highest reaching above $51{ }^{\circ} \mathrm{C}$. The vegetation of Cholistan desert is xeric, adapted to extremely high temperature, low moisture contents and increased salinity coupled with wide variation of edaphic factors. Main soil types of Cholistan desert are sand dunes (44\%), sandy soils (37\%), loamy soils (2\%) and saline-sodic clayey soils (17\%). Fortunately, several nutritious and drought tolerant species of grasses, shrubs and trees are found naturally in this desert especially after rainfall (Akbar et al., 1996).

\section{Reconnaissance survey and study sites}

A reconnaissance survey was conducted in January 2011, in order to have an impression of site conditions, to collect information about accessibility, to do an overview of plant assemblages and to determine the sampling and data collection methods. According to schedule, whole research project was carried for two consecutive years i.e. 2011 and 2012. After going through the topographic map of area followed by frequent visits during initial stages of study, research area was divided into 20 stands to cover the variations of physiognomy and physiography almost over the whole Cholistan desert. The specific stand position was determined by a GPS (Global Positioning System) named Garmin eTrex. The geographic coordinate's latitude, longitude, and altitude were taken from each stand (Table 1). 


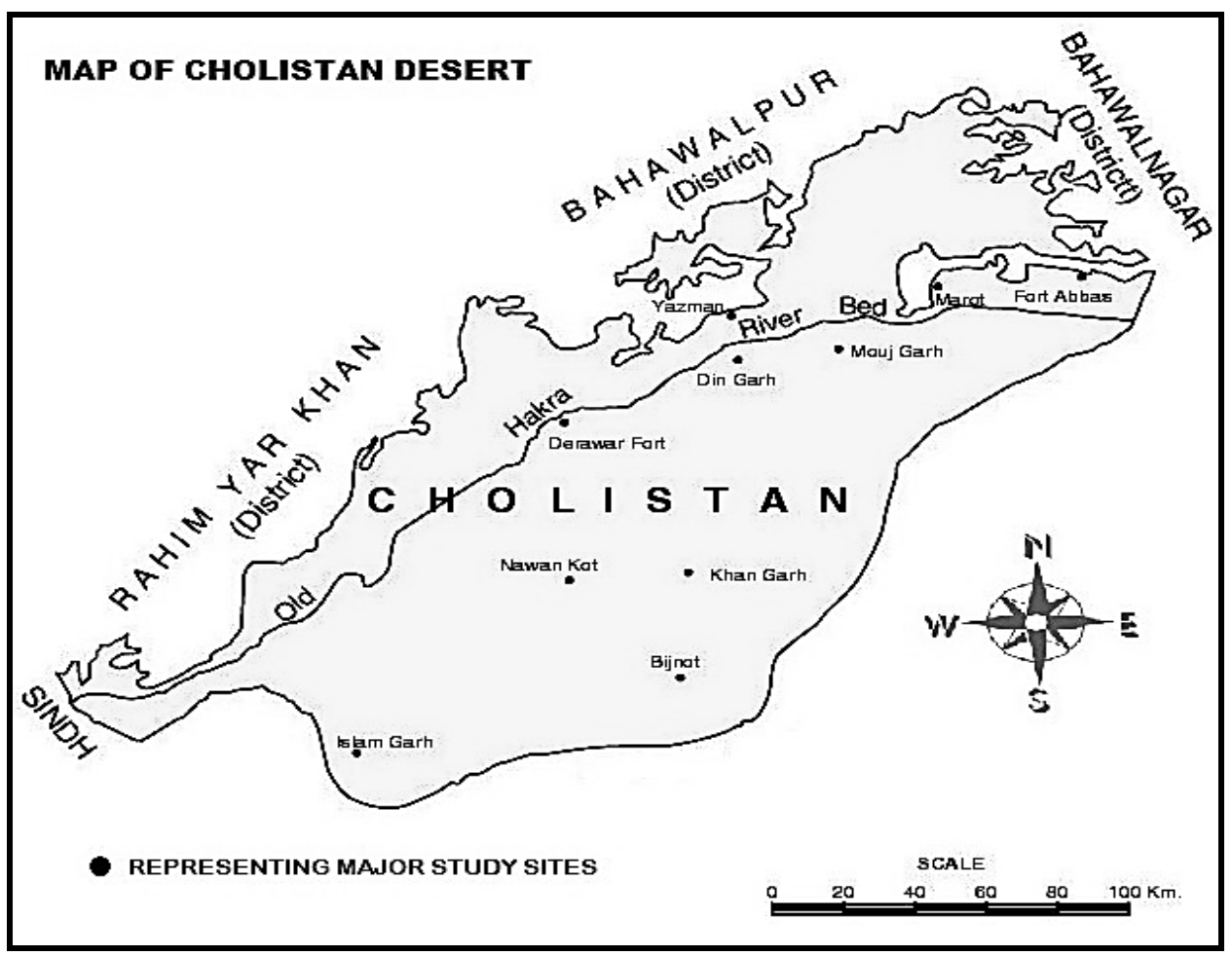

Figure 1. Map of study area; the Cholistan desert of Pakistan

Table 1. Name, location, and topography of each stand in study area

\begin{tabular}{|c|c|c|c|c|c|}
\hline Sr. No. & Stand Name & GPS Location & & Elevation & Topography \\
\hline 1 & Marot & $\mathrm{N}: 29^{\circ} 12.161^{\prime}$ & E: $072^{\circ} 15.427^{\prime}$ & $398 \mathrm{ft}$ & Sandunal \\
\hline 2 & Kalapahar & $\mathrm{N}: 29^{\circ} 10.430^{\prime}$ & E: $072^{\circ} 05.569^{\prime}$ & $384 \mathrm{ft}$ & Clayey saline \\
\hline 3 & Sulleh Wala & $\mathrm{N}: 28^{\circ} 40.315^{\prime}$ & E: $071^{\circ} 35.648^{\prime}$ & $389 \mathrm{ft}$ & Interdunal sandy \\
\hline 4 & Januwali & $\mathrm{N}: 29^{\circ} 05.056^{\prime}$ & E: $072^{\circ} 09.933^{\prime}$ & $406 \mathrm{ft}$ & Interdunal sandy \\
\hline 5 & Khirsir & $\mathrm{N}: 29^{\circ} 10.339^{\prime}$ & E: $072^{\circ} 08.749^{\prime}$ & $391 \mathrm{ft}$ & Sandunal \\
\hline 6 & Haider wali & $\mathrm{N}: 29^{\circ} 02.672^{\prime}$ & E: $072^{\circ} 10.200^{\prime}$ & $382 \mathrm{ft}$ & Clayey saline \\
\hline 7 & Mojgarh Fort & $\mathrm{N}: 29^{\circ} 01.059^{\prime}$ & E: $072^{\circ} 08.106^{\prime}$ & $392 \mathrm{ft}$ & Sandunal \\
\hline 8 & Khangarh & $\mathrm{N}: 28^{\circ} 57.261^{\prime}$ & E: $072^{\circ} 03.089^{\prime}$ & $369 \mathrm{ft}$ & Interdunal sandy \\
\hline 9 & Khanser & $\mathrm{N}: 28^{\circ} 59.227^{\prime}$ & E: $071^{\circ} 55.299^{\prime}$ & $352 \mathrm{ft}$ & Sandunal \\
\hline 10 & Bijnot & $\mathrm{N}: 28^{\circ} 47.988^{\prime}$ & E: $071^{\circ} 45.770^{\prime}$ & $340 \mathrm{ft}$ & Interdunal sandy \\
\hline 11 & Dingarh Fort & $\mathrm{N}: 28^{\circ} 57.454^{\prime}$ & E: $071^{\circ} 51.910^{\prime}$ & $365 \mathrm{ft}$ & Clayey saline \\
\hline 12 & Rukanpur & $\mathrm{N}: 28^{\circ} 53.182^{\prime}$ & E: $071^{\circ} 46.362^{\prime}$ & $371 \mathrm{ft}$ & Sandunal \\
\hline 13 & Nidamwala Toba & $\mathrm{N}: 28^{\circ} 52.963^{\prime}$ & E: $071^{\circ} 44.270^{\prime}$ & $355 \mathrm{ft}$ & Clayey saline \\
\hline 14 & Nawankot & $\mathrm{N}: 28^{\circ} 47.939^{\prime}$ & E: $071^{\circ} 45.770^{\prime}$ & $334 \mathrm{ft}$ & Interdunal sandy \\
\hline 15 & Lakhan & $\mathrm{N}: 28^{\circ} 52.232^{\prime}$ & E: $071^{\circ} 42.731^{\prime}$ & $351 \mathrm{ft}$ & Clayey saline \\
\hline 16 & Chananpir & $\mathrm{N}: 28^{\circ} 56.832^{\prime}$ & E: $071^{\circ} 40.057^{\prime}$ & $353 \mathrm{ft}$ & Interdunal sandy \\
\hline 17 & Baylawala & $\mathrm{N}: 29^{\circ} 23.466^{\prime}$ & E: $071^{\circ} 39.563^{\prime}$ & $410 \mathrm{ft}$ & Interdunal sandy \\
\hline 18 & Derawar fort & $\mathrm{N}: 29^{\circ} 23.465^{\prime}$ & E: $071^{\circ} 39.560^{\prime}$ & $345 \mathrm{ft}$ & Interdunal sandy \\
\hline 19 & Chasma Dhar & $\mathrm{N}: 28^{\circ} 39.864^{\prime}$ & E: $071^{\circ} 15.632^{\prime}$ & $323 \mathrm{ft}$ & Clayey saline \\
\hline 20 & Islamgarh Fort & $\mathrm{N}: 27^{\circ} 50.208^{\prime}$ & E: $071^{\circ} 48.129^{\prime}$ & $334 \mathrm{ft}$ & Sandunal \\
\hline
\end{tabular}




\section{Browse forage production}

Biomass is a commonly measured vegetation attribute that refers to weight of plant material within an area. Only plants that are available and palatable to grazing animals are classified as forage. Data was collected in wet (August) and dry season (April) both. Biomass was calculated by Direct Harvest method using 100-meter line transect with $1 \mathrm{x} 1$ meter square quadrate. At each stand five transect were laid out and quadrate were placed systematically at 10-meter interval on each transect. Clipping was done at grazed-height, because it gives more pertinent measure of forage biomass. The harvested material was packed and labeled in paper bags immediately and weighed in the field to get fresh weight and then oven dried at $65^{\circ} \mathrm{C}$ for 72 hours in laboratory and re weighed. The dry weight of all quadrates was then combined and averaged to get total dry matter production ( $\mathrm{kg} / \mathrm{ha})$, at each stand. Grazing status was estimated by direct observation at each stand and categorizes them as overgrazed, moderately grazed, slightly grazed and no grazing (Bonham, 1989; Holechek et al., 1995).

\section{Range carrying capacity}

Carrying capacity is an important management tool that connects forage supply with forage consumption. Carrying capacity was estimated based on $40 \%$ allowable grazing material. The one animal unit (AU) was taken as, a cow having $350 \mathrm{~kg}$ weight, demanding $7 \mathrm{~kg}$ dry matter forage per day, $2555 \mathrm{~kg} / \mathrm{year}$ (Bonham, 1989).

$$
\text { Carrying capacity }(\mathrm{ha} / \mathrm{AU} / \mathrm{Year})=\frac{\text { Animal forage requirement } \mathrm{kg} / \mathrm{year}}{\text { Forage production } \mathrm{kg} / \mathrm{ha}}
$$

\section{Degree of palatability}

Classification of browse species based on palatability, parts used and animal's preferences was recorded by direct observing the grazing livestock (cattle, sheep, goats and camel) in field for two consecutive years. These field observations were further confirmed from knowledge gathered from graziers and nomadic peoples at different range sites of Cholistan desert. In order to calculate the degree of palatability, following palatability classes were used:

i) Highly palatable ii) Moderately palatable iii) Less palatable iv) Non-palatable

The palatable species were classified into four categories based on parts used by livestock:

i) Leave grazed ii) Shoot grazed iii) Flower grazed and iv) Fruit grazed

The livestock mostly differ in their selection of browsing species at different range sites. In present case, browsing species were classified whether grazed by cattle, sheep, goat, or camel (Hussain and Durrani, 2009).

\section{Statistical analysis}

Microsoft Excel spreadsheet analysis (MS OFICE, 2010) was conducted to determine simple averages, percentiles and mean values and to make needful tables and graphs (McCullough and Heiser, 2008). 


\section{Results}

\section{Forage production of browses}

Browse forage production was estimated during wet (August) and dry season (April) in the arid rangelands of Cholistan desert. During wet season, total fresh browse productivity from all stands was $14034.6 \mathrm{~kg} / \mathrm{ha}$ and dry forage productivity was 8029.1 $\mathrm{kg} / \mathrm{ha}$. The highest quantity of dry phytomass was attained at stand $8(554.4 \mathrm{~kg} / \mathrm{ha})$ and minimum at stand $20(321.5 \mathrm{~kg} / \mathrm{ha})$. The average fresh forage yield recorded from all stand was $701.73 \mathrm{~kg} / \mathrm{ha}$ and dry forage yield was $401.46 \mathrm{~kg} / \mathrm{ha}$. On the basis of habitats, maximum dry matter production $(3681.3 \mathrm{~kg} / \mathrm{ha})$ was observed at interdunal habitat, followed by sandunal habitat $(2298.5 \mathrm{~kg} / \mathrm{ha})$ and then clayey saline habitat $(2049.3$ $\mathrm{kg} / \mathrm{ha}$ ). However, average dry forage production of browses at interdunal habitat was $460.16 \mathrm{~kg} / \mathrm{ha}$ followed by sandunal habitat with $383.08 \mathrm{~kg} / \mathrm{ha}$ then clayey saline habitat with $341.55 \mathrm{~kg} / \mathrm{ha}$ (Fig. 2). Based on grazing intensity in wet season, maximum stands $(45 \%)$ were observed to be moderately grazed followed by overgrazed (40\%) and then slightly grazed (15\%) (Table 2).

In Cholistan rangelands during dry season the total fresh biomass productivity was $8865.8 \mathrm{~kg} / \mathrm{ha}$ while dry matter production was $5422.9 \mathrm{~kg} / \mathrm{ha}$. The average fresh productivity of all stands was $443.29 \mathrm{~kg} / \mathrm{ha}$ and dry matter was $271.145 \mathrm{~kg} / \mathrm{ha}$. The maximum dry matter of browses was attained at stand $4(376.5 \mathrm{~kg} / \mathrm{ha})$ while minimum was at stand $20(161 \mathrm{~kg} / \mathrm{ha})$. Along habitats, maximum dry biomass was recorded at interdunal sandy habitat $(2633 \mathrm{~kg} / \mathrm{ha})$ followed by sandunal habitat $(1496.5 \mathrm{~kg} / \mathrm{ha})$ and then at clayey saline habitat $(1293.4 \mathrm{~kg} / \mathrm{ha})$. The average dry forage productivity of interdunal habitat was $329.13 \mathrm{~kg} / \mathrm{ha}$ followed by sandunal habitat with $249.42 \mathrm{~kg} / \mathrm{ha}$ then clayey saline habitat with $215.57 \mathrm{~kg} / \mathrm{ha}$ (Fig. 2). In this season, maximum stands were observed to be over grazed (75\%), followed by moderately grazed $(25 \%)$ and there was no stand without grazing effects (Table 2).

The comparison of vegetation condition during wet and dry seasons has been shown in Figure 3.

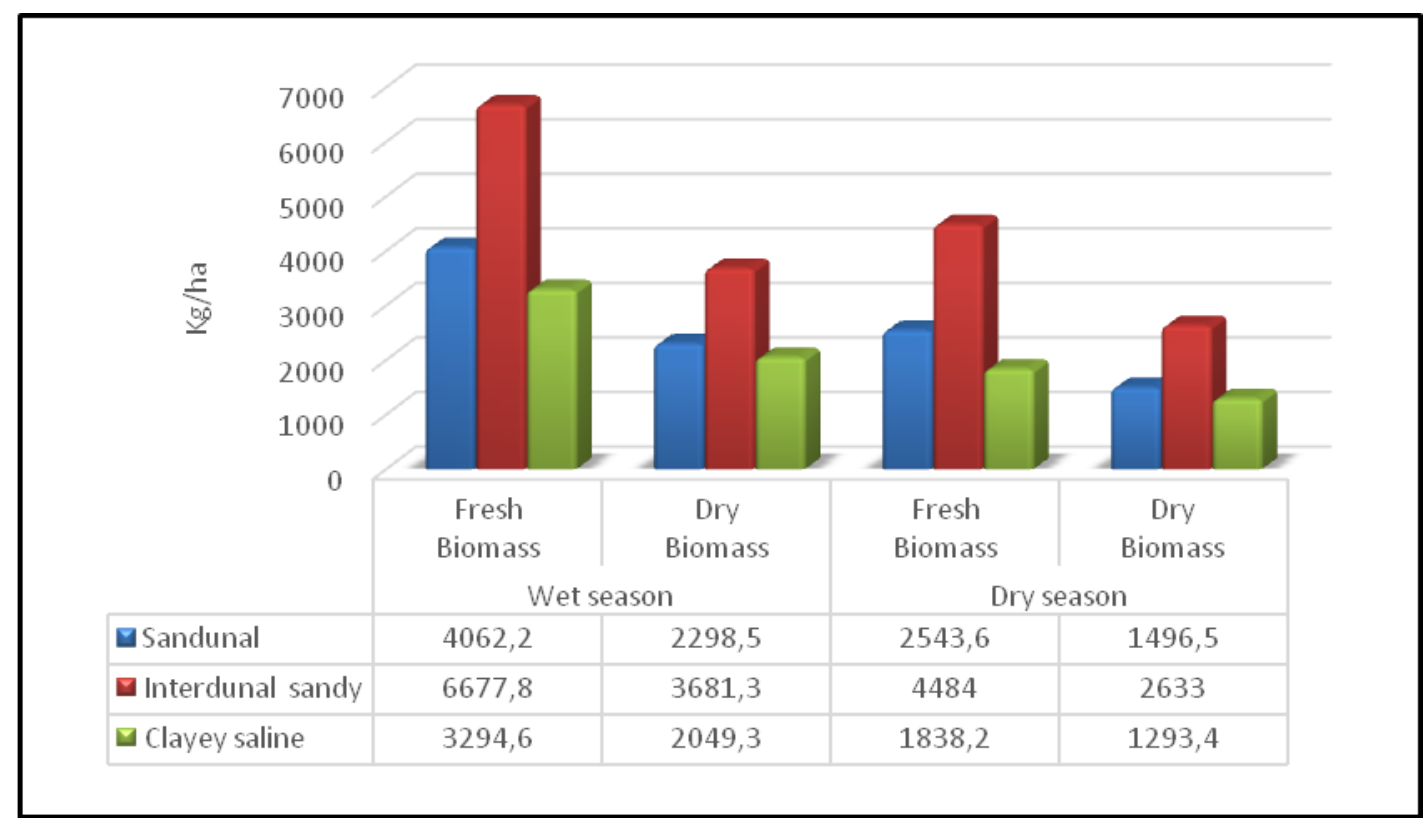

Figure 2. Seasonal browse forage yield $(\mathrm{kg} / \mathrm{ha})$ at three range habitats in Cholistan 
Table 2. Seasonal browse production $(\mathrm{kg} / \mathrm{ha})$ and grazing status of each stands

\begin{tabular}{|c|c|c|c|c|c|}
\hline Stand No. & Topography/Habitat & Season & Fresh biomass & Dry biomass & Grazing status \\
\hline \multirow[t]{2}{*}{1} & Sandunal area & Wet & 669.1 & 388.2 & Overgrazed \\
\hline & & Dry & 466 & 284.8 & Overgrazed \\
\hline \multirow[t]{2}{*}{2} & Clayey saline area & Wet & 517.4 & 328.4 & Overgrazed \\
\hline & & Dry & 344 & 221.8 & Overgrazed \\
\hline \multirow[t]{2}{*}{3} & Interdunal sandy area & Wet & 793.2 & 451.2 & Moderately grazed \\
\hline & & Dry & 577.8 & 322.5 & Overgrazed \\
\hline \multirow[t]{2}{*}{4} & Interdunal sandy area & Wet & 825.6 & 452.2 & Moderately grazed \\
\hline & & Dry & 582 & 376.5 & Moderately grazed \\
\hline \multirow[t]{2}{*}{5} & Sandunal area & Wet & 731.7 & 420.3 & Slightly grazed \\
\hline & & Dry & 436 & 244.8 & Moderately grazed \\
\hline \multirow[t]{2}{*}{6} & Clayey saline area & Wet & 623 & 347.7 & Moderately grazed \\
\hline & & Dry & 335.5 & 234.5 & Moderately grazed \\
\hline \multirow[t]{2}{*}{7} & Sandunal area & Wet & 690.3 & 365.2 & Moderately grazed \\
\hline & & Dry & 427.5 & 240 & Moderately grazed \\
\hline \multirow[t]{2}{*}{8} & Interdunal sandy area & Wet & 1017 & 554.4 & Slightly grazed \\
\hline & & Dry & 623 & 354.3 & Overgrazed \\
\hline \multirow[t]{2}{*}{9} & Sandunal area & Wet & 650.4 & 386.8 & Overgrazed \\
\hline & & Dry & 420.5 & 232.8 & Overgrazed \\
\hline \multirow[t]{2}{*}{10} & Interdunal sandy area & Wet & 920.3 & 523.7 & Slightly grazed \\
\hline & & Dry & 625.3 & 372.8 & Moderately grazed \\
\hline \multirow[t]{2}{*}{11} & Clayey saline area & Wet & 593.3 & 344.8 & Moderately grazed \\
\hline & & Dry & 327.5 & 255.5 & Overgrazed \\
\hline \multirow[t]{2}{*}{12} & Sandunal area & Wet & 628.6 & 376.9 & Overgrazed \\
\hline & & Dry & 402.3 & 240.8 & Overgrazed \\
\hline \multirow[t]{2}{*}{13} & Clayey saline area & Wet & 523.3 & 367.3 & Overgrazed \\
\hline & & Dry & 313.9 & 245.3 & Overgrazed \\
\hline \multirow[t]{2}{*}{14} & Interdunal sandy area & Wet & 810 & 495.6 & Moderately grazed \\
\hline & & Dry & 607.3 & 363.5 & Overgrazed \\
\hline \multirow[t]{2}{*}{15} & Clayey saline area & Wet & 570 & 339.6 & Moderately grazed \\
\hline & & Dry & 266.3 & 175.3 & Overgrazed \\
\hline \multirow[t]{2}{*}{16} & Interdunal sandy area & Wet & 737.4 & 391.4 & Overgrazed \\
\hline & & Dry & 493.8 & 285.3 & Overgrazed \\
\hline \multirow[t]{2}{*}{17} & Interdunal sandy area & Wet & 790.3 & 396.8 & Moderately grazed \\
\hline & & Dry & 489.3 & 257.8 & Overgrazed \\
\hline \multirow[t]{2}{*}{18} & Sandunal area & Wet & 692.1 & 361.1 & Moderately grazed \\
\hline & & Dry & 391.3 & 253.3 & Overgrazed \\
\hline \multirow[t]{2}{*}{19} & Interdunal sandy area & Wet & 784 & 416 & Overgrazed \\
\hline & & Dry & 485.5 & 300.3 & Overgrazed \\
\hline \multirow[t]{2}{*}{20} & Clayey saline area & Wet & 467.6 & 321.5 & Overgrazed \\
\hline & & Dry & 251 & 161 & Overgrazed \\
\hline
\end{tabular}


a

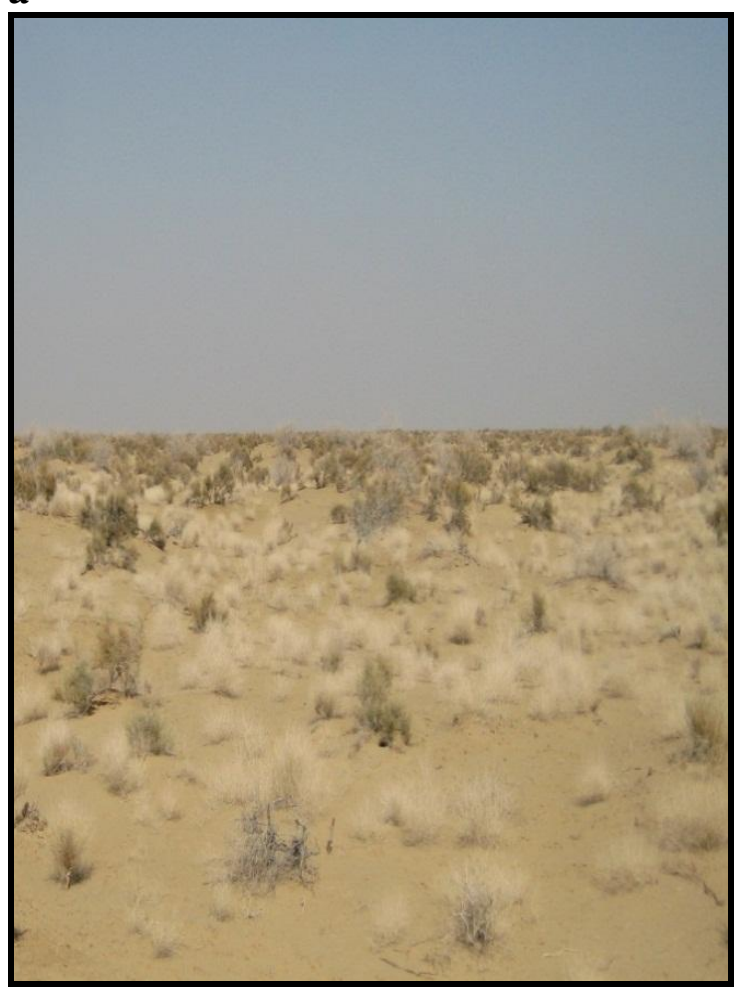

b

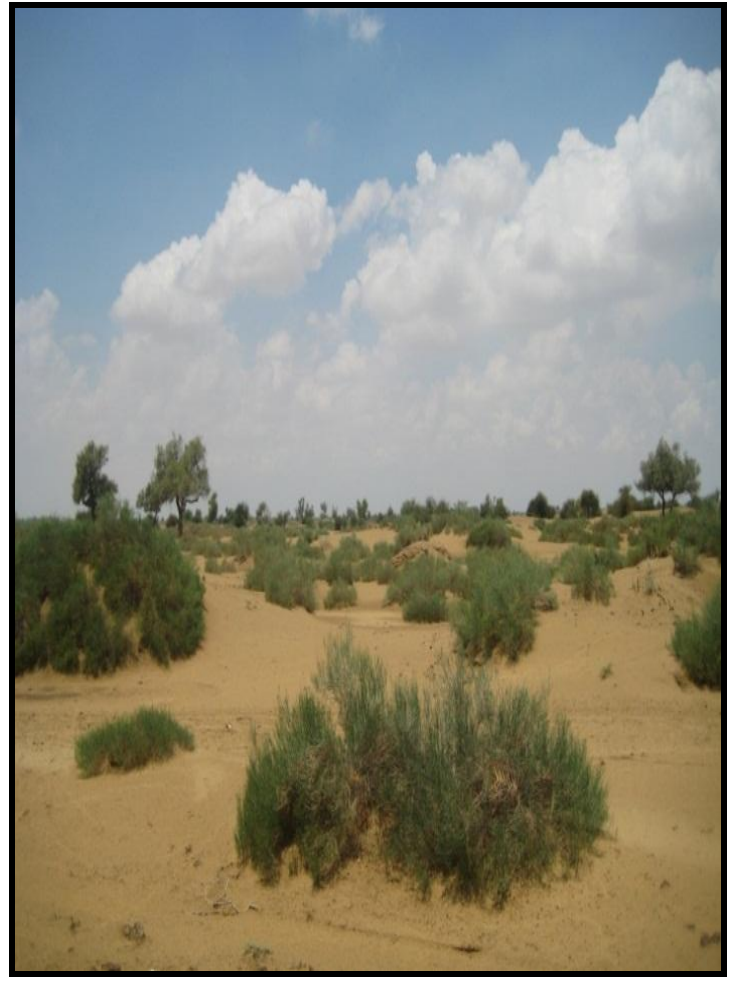

Figure 3. Vegetation condition in Cholistan desert during a-dry and b-wet seasons

\section{Range carrying capacity of browses}

Carrying capacity or grazing capacity is a common term, used when we are defining the stocking rates. In this study, carrying capacity was calculated in the rangelands of Cholistan desert with respect to browse production. As given in Table 3 overall average dry browse production during wet season was $394.93 \mathrm{~kg} / \mathrm{ha}$ while available forage production was $157.97 \mathrm{~kg} / \mathrm{ha}$. The proper use factor (PUF) has been taken as $40 \%$ to estimate available forage. Over three range habitats in Cholistan desert, maximum available forage was observed at interdunal sandy $(184.06 \mathrm{~kg} / \mathrm{ha})$ followed by sandunal $(153.23 \mathrm{~kg} / \mathrm{ha})$ and least at clayey saline $(136.62 \mathrm{~kg} / \mathrm{ha})$. However, carrying capacity (CC) during wet season was calculated as $16 \mathrm{ha} / \mathrm{AU} / \mathrm{Y}$ while at interdunal was 14/ha/AU/Y; at sandunal was $17 \mathrm{ha} / \mathrm{AU} / \mathrm{Y}$ and at clayey saline habitat was $19 \mathrm{ha} / \mathrm{AU} / \mathrm{Y}$. It was estimated that Cholistan desert cover an area of 2.6 million hectare out of which 1300000 ha has been considered as rangelands. Based upon this factor, the stocking rate during wet season was calculated as $80376 \mathrm{AU} / \mathrm{Y}$ in Cholistan rangelands.

In dry season, overall average dry matter production was $264.71 \mathrm{~kg} / \mathrm{ha}$ while the available forage was $105.88 \mathrm{~kg} / \mathrm{ha}$ (Table 3). Across the three range habitats in Cholistan desert, maximum available forage was recorded at interdunal habitat (131.65 $\mathrm{kg} / \mathrm{ha})$ followed by sandunal habitat $(99.77 \mathrm{~kg} / \mathrm{ha})$ and then at clayey saline habitat $(86.23 \mathrm{~kg} / \mathrm{ha})$. Based on available forage overall carrying capacity in dry season was calculated as $24 \mathrm{ha} / \mathrm{AU} / \mathrm{Y}$ whereas carrying capacity of interdunal was $19 \mathrm{ha} / \mathrm{AU} / \mathrm{Y}$, sandunal was $26 \mathrm{ha} / \mathrm{AU} / \mathrm{Y}$ and at clayey saline habitat was $30 \mathrm{ha} / \mathrm{AU} / \mathrm{Y}$. In this season, the stocking density of Cholistan rangelands was calculated as $53872 \mathrm{AU} / \mathrm{Y}$. 
Table 3. Seasonal carrying capacity (cc) at three range habitats in Cholistan

\begin{tabular}{lllll}
\hline Season & Range habitat & $\begin{array}{l}\text { Average browse production } \\
(\mathrm{kg} / \mathrm{ha})\end{array}$ & $\begin{array}{l}\text { Available forage } \\
(\mathrm{kg} / \mathrm{ha})\end{array}$ & $\begin{array}{l}\text { Carrying capacity } \\
(\mathrm{ha} / \mathrm{AU} / \mathrm{Y})\end{array}$ \\
\hline Wet season & Sandunal & 383.08 & 153.23 & 17 \\
& Interdunal sandy & 460.16 & 184.06 & 14 \\
& Clayey saline & 341.55 & 136.62 & 19 \\
& Overall average CC & 394.93 & 157.97 & 16 \\
Dry season & Sandunal & 249.42 & 99.77 & 26 \\
& Interdunal sandy & 329.13 & 131.65 & 19 \\
& Clayey saline & 215.57 & 86.23 & 30 \\
& Overall average. CC & 264.71 & 105.88 & 24 \\
\hline
\end{tabular}

\section{Palatability classification of browses}

Results regarding palatability of browses in Cholistan rangelands revealed that out of total species, $22(88 \%)$ species were found to have palatability to varying degree and $03(12 \%)$ species were found non-palatable. Among palatable species, 7 species $(31.82 \%)$ were highly palatable, 9 species $(40.91 \%)$ were moderately palatable, and 6 species $(27.27 \%)$ were less palatable. In highly palatable class, there were 5 species of trees and 2 species of shrubs. Moderately palatable species were consisting of 2 species of trees and 7 species of shrubs. Less palatable class was composed of 6 species of shrubs while non-palatable class was consisting of only 3 species of shrubs. There were total 7 species of trees in which $71.43 \%$ were highly palatable and $28.57 \%$ moderately palatable. Out of 18 species of shrubs, $11.11 \%$ species were highly palatable, $38.89 \%$ were moderately palatable, $33.33 \%$ were less palatable, and $16.67 \%$ species were unpalatable (Table 4).

Palatability by parts used was based on the type of plant parts, used by livestock in Cholistan rangelands. It was observed that among palatable species, leaves of 14 species (63.64\%), shoot/stem of 13 species (59.09\%), flower of 4 species (18.18\%), and fruit of 3 species $(13.64 \%)$ were grazed by livestock. The first class in which leaves were used, was consisting of 6 species of trees and 8 species of shrubs. In second class in which shoot/stem was used, there were 4 species of trees and 9 species of shrubs. Flower use class, was consisting of 2 species of trees and shrubs each. In fruit class, there were only 3 species of trees (Table 4).

According to results out of total palatable browse species, 7 species (31.82\%) were grazed by cattle, which comprised of 5 species of trees and 2 species of shrubs. Goats were observed to prefer 10 species $(45.45 \%)$ which consisted of 6 species of trees and 4 species of shrubs. Sheep grazed on 10 species $(45.45 \%)$ which were composed of 6 species of trees and 4 species of shrubs. Whereas, 20 species $(90.91 \%)$ were preferred by camel which consisting of 7 species of trees and 13 species of shrubs (Table 4). 
Table 4. Palatability classification of browse species in Cholistan rangelands

\begin{tabular}{|c|c|c|c|c|c|c|c|c|c|c|c|c|c|}
\hline \multirow[t]{2}{*}{ S.N. } & \multirow[t]{2}{*}{ Plant Species } & \multicolumn{4}{|c|}{$\begin{array}{l}\text { Degree of } \\
\text { palatability }\end{array}$} & \multicolumn{4}{|c|}{$\begin{array}{l}\text { Palatability } \\
\text { by parts used }\end{array}$} & \multicolumn{4}{|c|}{$\begin{array}{l}\text { Palatability by } \\
\text { livestock }\end{array}$} \\
\hline & & Нp & $\mathrm{Mp}$ & $\mathrm{Lp}$ & $\mathrm{Np}$ & $\mathrm{Lv}$ & Sh & $\mathrm{Fl}$ & $\mathrm{Fr}$ & $\mathrm{Ca}$ & Go & Sh & $\mathrm{Cm}$ \\
\hline 1 & Aerva javanica (Burm. f.) Merill. & - & - & - & + & - & - & - & - & - & - & - & - \\
\hline 2 & $\begin{array}{l}\text { Aerva pseudotomentosa ssp. bovei. } \\
\text { Clarke. }\end{array}$ & - & - & - & + & - & - & - & - & - & - & - & - \\
\hline 3 & Calotropis procera (Aiton.) Aiton. & - & - & + & - & + & - & - & - & + & + & + & - \\
\hline 4 & $\begin{array}{l}\text { Leptadenia pyrotecnica (Forssakal.) } \\
\text { Decne. }\end{array}$ & - & - & - & + & - & - & - & - & - & - & - & - \\
\hline 5 & Capparis decidua (Forsskal.) Edgew. & - & + & - & - & - & + & - & - & - & - & - & + \\
\hline 6 & Capparis spinosa Linn. & - & + & - & - & - & + & - & - & - & - & - & + \\
\hline 7 & Haloxylon recurvum Bunge. ex. Boiss. & - & + & - & - & - & + & - & - & - & - & - & + \\
\hline 8 & Haloxylon salicornicum (Moq.) Bunge. & - & - & + & - & - & + & - & - & - & - & - & + \\
\hline 9 & $\begin{array}{l}\text { Salsola baryosma (Roem. et. Scult.) } \\
\text { Dany. }\end{array}$ & - & + & - & - & - & + & - & - & - & - & - & + \\
\hline 10 & Suaeda fruticosa (Linn.) Farsskal. & - & + & - & - & + & + & - & - & - & - & - & + \\
\hline 11 & Pulicaria rajputanae Blatt. \& Hall. & - & - & + & - & + & - & - & - & - & - & - & + \\
\hline 12 & Abutilon muticum (Del. ex. DC.) Sweet. & - & - & + & - & + & - & - & - & - & - & - & + \\
\hline 13 & Acacia jacquemontii Benth. & + & - & - & - & + & + & + & - & - & + & + & + \\
\hline 14 & Acacia nilotica (Linn.) Del & + & - & - & - & + & + & + & + & + & + & + & + \\
\hline 15 & Prosopis cineraria (Linn.) Druce. & + & - & - & - & + & + & + & + & + & + & + & + \\
\hline 16 & Prosopis juliflora DC. & - & + & - & - & + & - & - & + & - & + & + & + \\
\hline 17 & Crotalaria burhia Ham. Ex. Bth. & - & - & + & - & + & - & - & - & - & - & - & + \\
\hline 18 & Tephrosia uniflora Pers. & - & - & + & - & + & - & - & - & - & + & + & - \\
\hline 19 & Calligonum polygonoides Linn. & - & + & - & - & - & + & + & - & - & - & - & + \\
\hline 20 & Zizyphus mauritiana Lam. & + & - & - & - & + & - & - & - & + & + & + & + \\
\hline 21 & $\begin{array}{l}\text { Zizyphus nummularia (Burm. f.) Wifht \& } \\
\text { Arn. }\end{array}$ & + & - & - & - & + & - & - & - & + & + & + & + \\
\hline 22 & Zizyphus spina christi (Linn.) Wild. & + & - & - & - & + & - & - & - & + & + & + & + \\
\hline 23 & Salvadora oleoides Decne. & + & - & - & - & + & + & - & - & + & + & + & + \\
\hline 24 & Tamarix aphylla (Linn.) Karst. & - & + & - & - & - & + & - & - & - & - & - & + \\
\hline 25 & Tamarix dioica Roxb. & - & + & - & - & - & + & - & - & - & - & - & + \\
\hline
\end{tabular}

Key words: Hp-Highly palatable, Mp-Moderately-palatable, Lp-Less palatable, Np-Non-palatable; Lv-Leaf, ShShoot, Fl-Flower, Fr-Fruit; Ca-Cattle, Go-Goat, Sh-Sheep, Cm-Camel

\section{Discussion}

\section{Forage production of browses}

The present study is endeavoring to assess biomass productivity of browse vegetation in Cholistan rangelands. According to results, total dry matter production during wet season was $8029.10 \mathrm{~kg} / \mathrm{ha}$ while in dry season was $5422.90 \mathrm{~kg} / \mathrm{ha}$, that was $19.38 \%$ higher in wet season. Whereas dry matter production at sandunal habitat was $21.14 \%$ high, at interdunal habitat was $16.6 \%$ high and at clayey saline habitat was $22.62 \%$ high in wet season as compare to dry season. Overall forage productivity was high during wet season across the three range habitats; this was perhaps due to high rainfall received 
during wet season as compared to dry season. Annual rainfall in Cholistan desert is extremely unpredictable both on temporal and spatial scales. Rainfall generally occurs during monsoon (July to September) and in winter and spring (January to March). The winter rain is scanty so vegetation in spring is usually poor, characterized by few annual species of forbs and grasses providing low biomass for grazing. In arid and semi-arid ecosystems, rainfall is a major environmental agent, affecting the forage productivity and it is extremely variable round the year (Patton et al., 2007). It was observed that Cholistan rangelands are monsoonal and forage productivity of these rangelands depends greatly on monsoon rain. Numerous studies have revealed that rainfall greatly affects the rangelands production and our conclusions agree with them (Farooq, 2003; Durrani and Hussain, 2005).

The rangelands in arid and semi-arid areas are mainly composed of perennial plants, which make the excellent use of climate and soil (Scasta and Rector, 2014). Based on results, it was observed that in both seasons dry forage production was high at interdunal habitat followed by sandunal and clayey saline habitats. This might be due to high vegetation diversity and better water retention capacity of soil at interdunal habitat. Vegetation coverage was low on sand dunes and unstable sand dunes were lacking vegetation. Whereas in clayey saline habitat soil and vegetation structure was poor, might be due to high $\mathrm{pH}$. Being very saline and impermeable to water the clayey saline habitat remained predominately plantless. It was observed that poor soil with less water holding capacity and low nutrients in Cholistan decrease the vigor and size of plants leading to reduced biomass (Katjiua and Ward, 2007).

Generally, difference in productivity level at selected range sites was due to variations in soil, vegetation type, and grazing pressure. Results showed that range sites with high forage productivity were less disturbed by grazing while sites with minimum forage productivity were mostly overgrazed. Both overgrazing and under grazing have adverse effects but overgrazing is more problematical (Gamoun, 2014). During wet season, maximum sites were moderately grazed while in dry season, maximum sites were overgrazed. There was no site without grazing in both seasons. In Cholistan rangelands, grazing period starts from August until February in good rainy years. About all forages were exploited during monsoon and post monsoon season, whereas some green browse remained available throughout year. The commencement of monsoon rains mostly commands the movement in nomadic peoples and livestock. In month of March or April, shortage of water and feed resources in interior of desert compel nomadic peoples and their herds to move towards irrigated plains (Akhtar and Arshad, 2006).

\section{Range carrying capacity of browses}

According to results overall browse productively was low in Cholistan rangelands whereas production was high in wet season as compare to dry season. As grazing animals in Cholistan rangelands comprised of cattle, sheep, goats, and camels therefore, carrying capacity was calculated for these kinds of animals. Holechek (1988) has determined daily dry matter (DM) intake for bighorn sheep, elk, moose, white-tailed deer, mule deer, and pronghorn antelopes as two percent of their body weight. As DM intake for the livestock of Cholistan rangelands has not been analyzed yet, thus DM intake for these animals was also taken as two percent of their live-body weight. Based on evidences of livestock producers of the area, a young cow (equal to one AU) may attain average live-body weight of about $350 \mathrm{~kg}$ whereas DM requirement of an AU was calculated as $7 \mathrm{~kg} \mathrm{ha}^{-1}$. 
Grazing time depends on availability, accessibility, and quality of feed which is reduced when quality forage is abundant (Khumalo et al., 2007). Based on USA recommendations, range utilization intensity is 30 to $40 \%$ of key species with 130 to $300 \mathrm{~mm}$ annual rainfall for shrub steppe in semiarid region. It may reach at $50 \%$ utilization level during high productive year and decrease during dry period (Holechek, 1988). In arid rangelands of Cholistan, range use intensity was taken as $40 \%$. Overall, available browse production during wet season was $157.97 \mathrm{~kg} / \mathrm{ha}$ and $105.88 \mathrm{~kg} / \mathrm{ha}$ during dry season. Based on these standards; overall carrying capacity during wet season was $16 \mathrm{ha} / \mathrm{AU} / \mathrm{Y}$ and in dry season was $24 \mathrm{ha} / \mathrm{AU} / \mathrm{Y}$. Whereas on browse forage availability, in wet season 80376 AU/Y while in dry season 53872 AU/Y were estimated that can be grazed in Cholistan rangelands.

Based on three range habitats in Cholistan, carrying capacity was high at interdunal habitat followed by sandunal and clayey saline habitats during the both seasons. Forage production was better in wet season than dry season (April to June) therefore, carrying capacity was high in wet season as compare to dry season. The data suggested that carrying capacity during both seasons was very low and available browse production was insufficient for present stocking rate (Guevara et al., 2009).

Similarly, nomadic peoples in Cholistan desert have also exploited the plant resources of this area. They uprooted almost every plant for their need irrespective of its forage, medicinal or other values. Therefore, forage productivity is decreasing day by day ultimately leading to poor carrying capacity. No doubt these rangelands are dominated by non-equilibrium condition. In this environment, drought will always be a key factor in affecting production level and animal numbers. Whereas, to decide grazing prescriptions a resource manager needs to define variations in productivity levels and provide estimates of stocking rates (Bisigato et al., 2005).

\section{Palatability classification of browses}

It was observed that identified browse species comprising 7 species of trees and 18 species of shrubs were almost remained available throughout the year in Cholistan rangelands. As rangelands vegetation varied significantly in their seasonal availability, nutritive value, and palatability, so grazing animals apparently select highly palatable forage species first (Heitschmidt et al., 2005). According to results, maximum browse species were found to be moderately palatable. However, seven species were highly palatable which consist of 5 species of trees (Acacia nilotica, Prosopis cineraria, Zizyphus mauritiana, Zizyphus spina Christi, Salvadora oleoides) and 2 species of shrubs (Acacia jacquemontii, Zizyphus nummularia). Whereas, three species were found to be unpalatable which consist of only shrubs including Aerva javanica, Aerva pseudotomentosa and Leptadenia pyrotechnica. This unpalatability might be due to alkaloids, phenolics, saponins and other poisonous elements. It is very difficult to differentiate between non-poisonous and poisonous plants as animal's dislike feed due to unlikable feelings or physical discomfort, or by excess or deficiency of nutrients (Kayani et al., 2007).

Animals greatly differ in their preferences for selection of various plant species or plant parts as feed. Results showed that maximum trees were used for their leaves, whereas maximum shrubs were preferred for their shoot. Leaves have been grazed in maximum browse species (63.64\%), as feed by grazing animals. The livestock usually prefer leaves of all forages, might be due to high crude protein, phosphorus and low lignin and fiber contents than woody parts. Generally, animals desire fresh foliages than 
dried and non-succulent forages that can be eaten easily. Likewise, soft green herbaceous parts, in addition having good taste and odour are rapidly digestible (Sanon et al., 2007). It was also observed that Acacia nilotica, Prosopis cineraria, and Prosopis juliflora were preferred for their fruits by livestock. Flowers and fruits are seasonally essential in animal feed as they might have high level of proteins and cell soluble than leaves. Fresh forage species with high contents of crude protein, sugar, cellulose and fats are highly preferred and digestible. While plant species with high lignin, fiber, silica, secondary metabolites and with poor digestibility are less preferred by grazing animals (Holechek et al., 1998).

Rangelands of Cholistan desert are freely grazed by mixed herds of cattle, sheep, goats and camels. According to results, camel ranked first in exploring maximum number of species, which consist of 7 species of trees, 13 species of shrubs. Goat and sheep were observed to have similar selection, which consisted of 6 species of trees and 4 species of shrubs. Cattle were observed to utilize minimum number of species including 5 species of trees and 2 species of shrubs. It was observed that grazing animals select the most palatable plant species first. It may lead to complete replacement of good quality forages by non-palatable species (Rutherford and Powrie, 2013). Animals face forage deficiency in Cholistan rangeland in winter (December and January) but this condition become severe in April owing to climatic extremities and shortage of water. Certain perennial browse species continue to maintain their foliage but deficiency of forage forces the animals to eat even less palatable species. It may be possible that poor health of animals in Cholistan rangelands are partially due to continuous utilization of such unpalatable species (Deng et al., 2013).

This study revealed that area was vegetative rich in wet season as compare to dry season. Browse species were found playing significant role in the provision of forage for livestock round the year particularly during droughts. The data suggested that carrying capacity during both seasons was very low and available browse production was insufficient for present stocking rate. Due to continuous grazing pressure, the palatable species were disappearing and unpalatable species were spreading on the landscape. It was observed that in Cholistan rangelands maximum forage was available during monsoon season because sustainability of life in this desert rotates round the annual precipitation. Numerous species of ephemeral and annual appear after rains, complete their life cycle in a short duration and vanish. These species, as well important in nutritional contribution also decrease grazing pressure on palatable perennial browse species (Gamoun et al., 2015).

\section{Conclusions}

This study has provided baseline about seasonal forage productivity, carrying capacity and palatability of browse vegetation in Cholistan rangelands. However, this study is very preliminary and it is recommended that subsequent ecological studies should be conducted on spatial and temporal variations about forage production. The productive potential of rangelands is not constant and carrying capacities need to be periodically reviewed to accommodate any changes in land resources, or environment. There is severe problem of overgrazing that leads to year-round stress on browse species. Grazing at suitable stocking rate is compulsory. Planned grazing should be introduced and implemented to release the stress over browse species. All factors considered, it was concluded that Cholistan rangelands are less productive and they 
need proper protection, management, and rehabilitation through ecological approaches. This data should be incorporated into the current management plan and subsequent vegetation map should serve as a valuable tool in planning, conservation and management of these rangelands.

Acknowledgements. Authors gratefully acknowledge the support from Higher Education Commission (HEC) Pakistan under indigenous scholarship program. Authors are also indebted to Late Dr. Muhammad Arshad (Ex. Director Cholistan Institute of Desert Studies) for his valuable supervision during the whole work.

\section{REFERENCE}

[1] Abdullah, M., Khan, R. A., Yaqoob, S., and Ahmad, M. (2013): Community structure of browse vegetation in Cholistan rangelands of Pakistan. - Pak. J. Agri. Sci. 50(2): 237-247.

[2] Ahmad, S. D., Hasnain, G. (2001): Evaluation of introduced fescue grass for forage and soil conservation in hilly areas of Pakistan. - Journal of Biological Sciences 1 (61): 442-445.

[3] Akbar, G., Khan, T. N., Arshad, M. (1996): Cholistan desert Pakistan. - Rangelands 18: 124-128.

[4] Akhter, R., Arshad. M. (2006): Arid Rangelands in Cholistan Desert Pakistan. - Scheresse 17: 1-18.

[5] Arshad, M., Hassan, A. U., Ashraf, M. Y., Noureen, S., and Moazzam. M. (2008): Edaphic factors and distribution of vegetation in the Cholistan desert, Pakistan. - Pak. J. Bot. 40: 1923-1931.

[6] Bisigato, A. J., Bertiller, M. B., Ares, J. O., Pazos, G. E. (2005): Effect of grazing on plant patterns in arid ecosystems of Patagonian Monte. - Ecography 28: 561-572.

[7] Bonham, C. D. (1989): Measurement for Terrestrial Vegetation. - John Wiley \& Sons. New York. pp. 199-264.

[8] Curtin, C. G. (2002): Livestock grazing, rest, and restoration in arid landscapes. Conserv. Biol. 16: 840-2.

[9] Deng, L., Sweeney, S., Shangguan, Z. P. (2013): Grassland responses to grazing disturbance:plant diversity changes with grazing intensity in a desert steppe. - Grass Forage Sci. doi:10.1111/ gfs.12065.

[10] Durrani, M. J., Hussain, F. (2005): Ethnoecological profile of plants of Harboi rangeland, Balochistan. - Int. J. Biol.\& Biotech. 2: 15-22.

[11] Farooq, M. U. (2003): Some suitable and sustainable strategies for improving rangeland productivity in Pakistan. - Pak. J. Forest 53: 193-199.

[12] Friedel, M. H., Laycock W. A., Bastin. G. N. (2000): Assessing Rangeland Condition and Trend. Field and Laboratory Methods for Grassland and Animal Production Research, CABI International, Wallingford, UK. pp. 305-360.

[13] Gamoun, M. (2014): Grazing intensity effects on the vegetation in desert rangelands of Southern Tunisia. - J Arid Land 6: 324-33.

[14] Gamoun, M., Bob, P., Hanchi, B. (2015): Assessment of vegetation response to grazing management in arid rangelands of southern Tunisia. - International Journal of Biodiversity Science, Ecosystem Services \& Management 11(2): 106-113.

[15] Guevara, J. C., Gru"nwaldt, E. G., Estevez, O. R., Bisigato, A. J., Blanco, L. J., Biurrun, F. N., Ferrando, C. A., Chirino, C. C., Morici, E., Ferna'ndez, B., Allegretti, L. I., Passera, C. B. (2009): Range and livestock production in the Monte Desert, Argentina. Journal of Arid Environments 73: 228-237.

[16] Heitschmidt, R. K., Klement, K. D., Haferkamp, M. R. (2005): Interactive effects of drought and grazing on Northern Great Plains Rangelands. Rangeland Ecol. Manage. 58: 11-19. 
[17] Holechek, J. L., Pieper, R. D., Herbel. C. H. (1995): Range management principles and practices. - Prentice Hall, Englewood Cliffs, NJ. 2nd ed. pp. 135-136.

[18] Holechek, J. L., Pieper, R. D., Herba, C. H. (1998): Range Management. Principles and practices. 3rd Edition. - Prentice Hall, Upper Saddle River, New Jersey, 07458.

[19] Holechek, J.L. (1988): An approach for setting the stocking rate. - Rangelands 10: 10-14.

[20] Hussain, F., Durrani, M. J. (2009): Seasonal availability, palaitability and animal preferences of forage plant in Harboi Arid Rangeland, Kalat, Pakistan. - Pak. J. Bot. 41(2): 539-554.

[21] Katjiua, M.,Ward, D. (2007): Pastoralists' perceptions and realities of vegetation change and brows consumption in the northern Kalahari, Namibia. - Journal of Arid Environments 69: 716-730.

[22] Kayani, S. A., Masood, A., Achakzai, A. K. K., Anbreen, S. (2007): Distribution of secondary metabolites in plants of Quetta-Balochistan. - Pak. J. Bot. 39: 1179-1179.

[23] Khan, Z. I., Valeem, E., Hussain, A., Ashraf, M., Ashraf, M. Y., McDowell, Akhtar, M.S., Mirza, M. A. (2005): Effect of seasonal variation on $\mathrm{Zn}$ content of soil, forage, water, feed and small ruminants grazing the native and improved pasture during different seasons in the semi-arid regions of Pakistan. - Int. J. Biol. Biotechnol. 2: 465-74.

[24] Khumalo, G., Holechek, J., Thomas, M., Molinar, F. (2007): Long-term vegetation productivity and trend under two stocking levels on Chihuahuan desert rangeland. Rangeland Ecol. Manage. 60: 165-171.

[25] Landsberg, J., Crowley, G. (2004): Monitoring rangeland biodiversity: Plants as indicators. - Austral Ecol. 29: 59-77.

[26] Loeser, M. R., Sisk, T. D., Crews, T. E. (2006): Impact of grazing intensity during drought in an Arizona Grassland. - Conserv. Biol. 21: 87-97.

[27] Majeed, A., Azam, M., Mumtaz, A. (2002): Drought and Water Management Strategies in Pakistan. Proceedings of the SAARC Workshop on Drought and Water Management Strategies (September 16-18, 2002 Lahore-Pakistan). - Pakistan Council of Research in Water Resources, Islamabad, Pakistan.

[28] McCullough, B. D., Heiser, D. A. (2008): On the accuracy of statistical procedures in Microsoft Excel 2007, Computational Statistics and Data Analysis. 52: 4570-4578.

[29] Mtengeti, E. J., Mhelela, A. (2006): Screening of potential indigenous browse species in semi-arid central Tanzania. A case of Gairo division. - Livestock Research for Rural development 18: 9.

[30] Patton, B. D., Dong, X. J., Nyren, P. E., Nyren, A. (2007): Effects of grazing intensity, precipitation, and temperature on forage production. - Rangeland Ecol. Manag. 60: 656665.

[31] Rubanza, C. D. K., Shem, M. N., Ichinohe, T., Fujihara, T. (2006): Biomass production and nutritive potential of conserved forages in silvo pastoral traditional fodder banks (Ngitiri) of Meatu District, Tanzania. Asian- Australians. - J. Anim. Sci. 19: 978-983.

[32] Rutherford, M. C., Powrie, L. W. (2013): Impacts of heavy grazing on plant species richness: a comparison across rangeland biomes of South Africa. - South Afr. J. Bot. 87:146-156.

[33] Sanon, H. O., Kabore'-Zoungrana, C., Ledin, I. (2007): Behaviour of goats, sheep and cattle and their selection of browse species on natural pasture in a Sahelian area. - Small Rumin. Res. 67: 64-74.

[34] Scasta, J. D., Rector, B. S. (2014): Drought and ecological site interaction on plant composition of a semi-arid rangeland. - Arid Land Res Manag. 28: 197-215. 